\title{
A NEW CALIBRATION METHOD FOR LOW COST MEMS INERTIAL SENSOR MODULE
}

Sheng-Chih Shen

Department of Systems and Naval Mechatronic Engineering, National Cheng Kung University, Tainan 701, Taiwan, R.O.C., scshen@mail.ncku.edu.tw

Chia-Jung Chen

Department of Systems and Naval Mechatronic Engineering, National Cheng Kung University, Tainan 701, Taiwan, R.O.C

Hsin-Jung Huang Department of Systems and Naval Mechatronic Engineering, National Cheng Kung University, Tainan 701, Taiwan, R.O.C

Follow this and additional works at: https://jmstt.ntou.edu.tw/journal

Part of the Electrical and Computer Engineering Commons

\section{Recommended Citation}

Shen, Sheng-Chih; Chen, Chia-Jung; and Huang, Hsin-Jung (2010) "A NEW CALIBRATION METHOD FOR LOW COST MEMS INERTIAL SENSOR MODULE," Journal of Marine Science and Technology. Vol. 18: Iss. 6, Article 5.

DOI: $10.51400 / 2709-6998.1939$

Available at: https://jmstt.ntou.edu.tw/journal/vol18/iss6/5

This Research Article is brought to you for free and open access by Journal of Marine Science and Technology. It has been accepted for inclusion in Journal of Marine Science and Technology by an authorized editor of Journal of Marine Science and Technology. 


\section{A NEW CALIBRATION METHOD FOR LOW COST MEMS INERTIAL SENSOR}

MODULE

\section{Acknowledgements}

The authors would like to thank National Science Council (NSC) for their financial supports to the project (granted number: NSC 97-2628-E-006-124-MY2). 


\title{
A NEW CALIBRATION METHOD FOR LOW COST MEMS INERTIAL SENSOR MODULE
}

\author{
Sheng-Chih Shen*, Chia-Jung Chen*, and Hsin-Jung Huang*
}

Key words: MEMS, inertial sensors, navigation, underwater.

\begin{abstract}
This paper presents a new calibration method to overcome the challenges of MEMS inertial sensors for underwater navigation. The MEMS inertial sensor module is composed of an accelerometer, a gyroscope and a circuit of signal process. For navigation estimation, it is easy to be influenced by errors which come from MEMS inertial sensors. In general, the sources of error can be categorized into two groups, deterministic type and stochastic type. The former primarily includes bias errors, misalignment and nonlinearity; the latter contains temperature effect and signal drifting. Subsequently, the linearity calibration is used to modify the deterministic error and the wavelet analysis can suppress the stochastic noise. Therefore, the new calibration method integrated of linearity calibration and wavelet signal processing is utilized to enhance the accuracy and performance of MEMS inertial sensor module. The experimental results demonstrate that the output signal can be corrected suitability by means of the proposed method.
\end{abstract}

\section{INTRODUCTION}

In recent years, the rapid development in micro-electromechanical system (MEMS) has capability to fabricate cheap, lightweight and low-power chip-based accelerometers and gyroscopes, which have been adopted into various applications such as navigation or positioning, consumer electronics, medical electronics [2,18]. Although the MEMS inertial sensors have such advantages mentioned above, they are still suffering from large errors including bias error, nonlinearity, misalignment, random noise, temperature effect $[12,14]$. Most MEMS inertial sensors are comb-drive vibratory mechanical structures fabricated on silicon wafer. Common fabrication processes include surface micromachining, bulk micromachining, high aspect ratio micromachining, and wafer-to-wafer bonding. Each of these fabrication steps involves multiple processes such as deposition, etching and patterning of materials. In

Paper submitted 05/21/09; revised 03/03/10; accepted 03/24/10. Author for correspondence: Sheng-Chih Shen (e-mail: scshen@mail.ncku.edu.tw).

*Department of Systems and Naval Mechatronic Engineering, National Cheng Kung University, Tainan 701, Taiwan, R.O.C. practical, every fabrication process contributes to defects in the MEMS inertial sensors [16]. Fabrication defects that cause asymmetric structures, misalignment of actuation mechanism, and deviations of the center of mass from the geometric center, result in undesirable systematic perturbations in the form of mechanical and electrostatic forces, which degrade the performance of MEMS inertial sensors. It is no doubt that the performance will be seriously degraded without a calibration. Hence, it is essential to apply different techniques to developing an effective calibration method to increase the precision and stability.

For the navigation system employing MEMS inertial sensors, several estimation techniques are chosen currently to eliminate noise problems. For example, the Kalman filter is widely utilized to overcome uncertainty problem due to errors which come from inertial sensors $[8,9,11,13]$. In addition, fuzzy modeling is used to approximate the navigation trajectory as precise as possible [7]. On the other hand, artificial neural networks are also utilized to recover the original signals from contaminated output signals [6]. Furthermore, some specific approaches for the effect of stochastic noise for the MEMS inertial sensors are also discussed. In [1], the temperature variation effects on MEMS inertial sensors are considered through two methods of thermal testing. In [5], the variation of temperature on noise models for MEMS inertial sensors is examined by using different statistics techniques such as Allan variance and least-squares spectral analysis (LSSA). And in [17], the random drift of MEMS gyroscope is modeled based on neural network which is built with radial basis network structure. However, for the underwater navigation or positioning, the situation is more complicated when AUV or ROV is moving in the water. One of the key difficulties in underwater navigation or positioning is that MEMS inertial sensors will be interfered with many environmental factors such as vibration, pressure and temperature. Basically, it is not simple to correct output signal for lack of external reference signal, especially in such a harsh environment. Therefore, it is desirable to use alternative ways to treat those MEMS inertial sensors. In this paper, an integrated calibration is presented as pre-processing for the sake of refining from raw inertial data. The MEMS inertial sensors can be calibrated properly to enhance the precision and performance effectively. Detailed information regarding the performance of the calibration method is presented in this paper. 


\section{THE ANALYSIS OF MEMS INERTIAL SENSORS}

\section{Sensor Error Model}

MEMS inertial sensor module, consisting of accelerometers and gyroscopes, is one of the most important CMOS-MEMS sensors. Basically, an accelerometer is a device that can sense force according to Newton's second law of motion. It is composed of a seismic mass and a capacitance to voltage converting circuit [3]. When there is any force acting on the accelerometer, the seismic mass would deflect and cause the change in capacitance, as well as in voltage. And the microstructure of accelerometer from our Lab is shown in Fig. 1.

In contrast, the operating principle of a gyroscope is based on the Coriolis effect. It consists of vibrating mechanical elements to sense rotation and converts it into an electrical signal output. In brief, the MEMS inertial sensor module outputs a voltage proportional to the physical quantities sensed by the sensors, acceleration and angular rates, respectively. Nevertheless, the actual relationship between the output voltage and the physical quantity acting along the sensor sensitivity axes is not consistent in the presence of errors, as shown in Fig. 2. These error characteristics should be described to provide a concise viewpoint for calibration. Normally, there is often a non-zero output called bias or zero offset in the sensor output signal when there is no force acting onto the sensor. Moreover, the relationship between the output signal of the accelerometer (gyro) and measured force (angular rate) is not necessary linear; i.e., nonlinearity error should be considered in practice. Even if the deterministic errors can be determined through a series of lab tests, but the stochastic noise is hard to be removed from the data using deterministic models. It is supposed to be treated as a random process. While the misalignment is negligible, the actual output of the MEMS inertial sensors module can be expressed as

$$
y_{\text {meas }}=(1+s) u_{\text {real }}+b_{\text {meas }}+n
$$

where $y_{\text {meas }}$ is the measured output of sensor, $s$ is the scale factor, $u_{\text {real }}$ is the ideal input, the $b_{\text {meas }}$ is the bias and $n$ represents stochastic noise. As indicated by (1), the bias and the scale factor are the main concerns for the deterministic error sources and the last term is the stochastic variation of the sensor output, which is owing to Brownian motion of gas molecules surrounding the seismic mass. The thermal noise equivalent acceleration (TNEA) is shown as [4]

$$
T N E A=\sqrt{\frac{4 K_{B} T \omega}{M Q}}
$$

where $K_{B}$ is the Boltzmann constant, $T$ is the absolute temperature, $\omega$ is the resonant frequency, $M$ is the weight of the seismic mass and $Q$ is the quality factor.

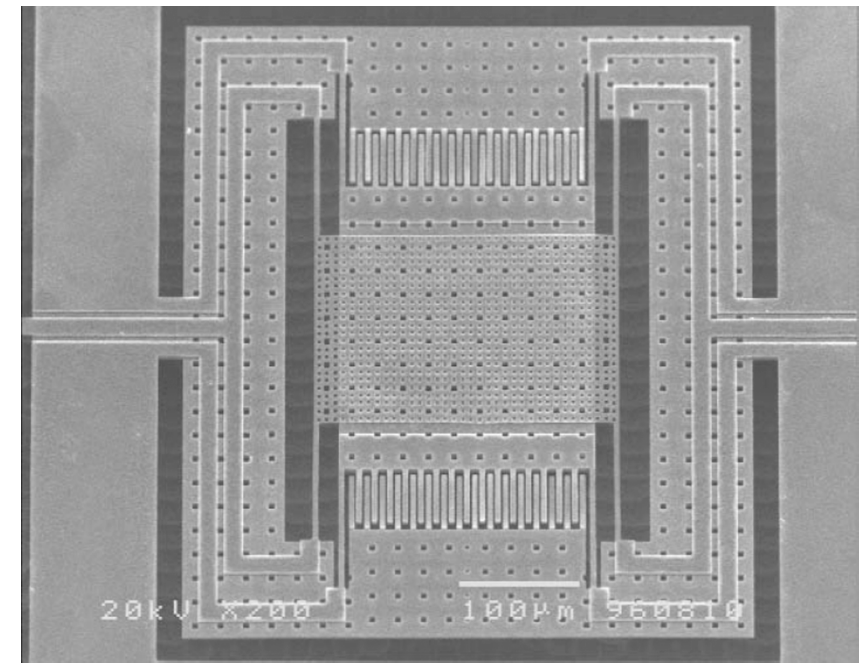

Fig. 1. The microstructure of accelerometer.

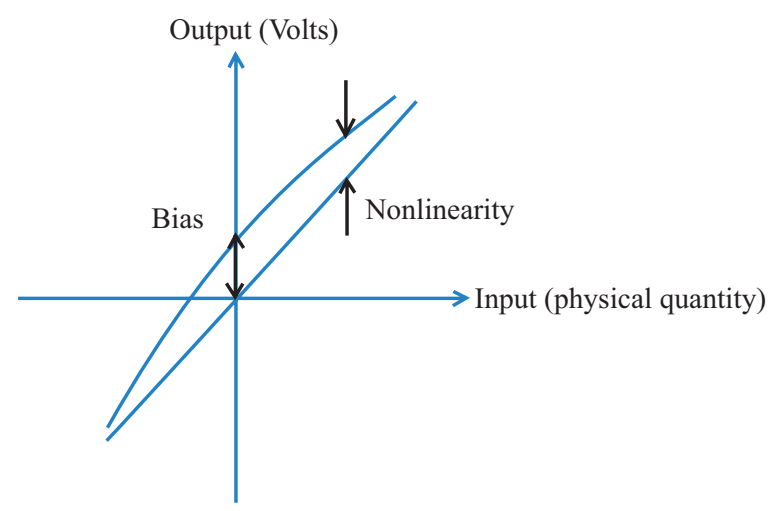

Fig. 2. The model of the deterministic errors.

\section{Proposed Calibration Method}

According to our sensor error model depicted above, the proposed calibration method is composed of three portions, bias compensation, linearity calibration and wavelet denoising. The bias for MEMS inertial sensor is the average signal which has no relation with the input quantity sensed by the sensors. That is, the sensors produce a nonzero output when there is no force acting onto them. Bias usually can be viewed as a static measurement in the long term, but it may still vary with time and temperature randomly. As for scale factor, the linearity calibration is performed by using a turntable to correct data with different angles for accelerometer or angular rates for gyro, and then the ideal characteristic equation can be obtained by recursive least squares (RLS) algorithm [10]. This algorithm is shown as follows:

$$
\begin{gathered}
\hat{x}_{N+1}=\hat{x}_{N}+K_{N+1}\left(y_{N+1}-\Phi^{T} \hat{x}_{N}\right) \\
K_{N+1}=P_{N} \Phi_{N+1}\left(\lambda+\Phi_{N+1}^{T} P_{N} \Phi_{N+1}\right)^{-1}
\end{gathered}
$$




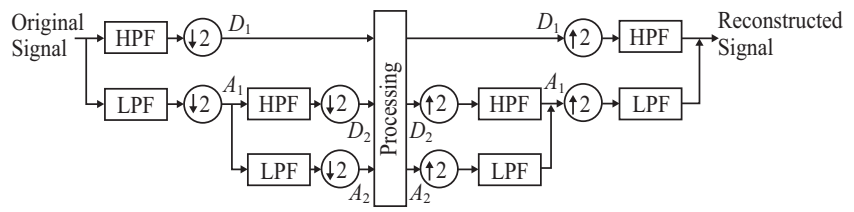

Fig. 3. The architecture of the wavelet analysis.

$$
P_{N+1}=\frac{1}{\lambda}\left(I-K_{N+1} \Phi_{N+1}\right)^{-1} P_{N}
$$

where $\Phi$ is the undetermined coefficient matrix, $y$ is the measurement matrix, $x$ is the desire input matrix and $\lambda$ is the forgetting factor which is recommended to choose from 0.95 to 0.98 for engineering applications.

Although the deterministic errors can be eliminated efficiently, there are some drawbacks that make MEMS inertial sensors unsuitable for real-time application. To be more specific, the output signal is always nonstationary, which means it is difficult to predict the output signal each time. As a useful filtering technique, wavelet analysis has become a powerful tool of signal analysis during the last two decades and widely used in many applications including signal detection and denoising, image processing, pattern recognition, medical diagnostics, etc. [15]. Theoretically, it provides a time-frequency representation of the signal without changing the information content present in the signal. The wavelet analysis is done similar as STFT (short-time Fourier transform) analysis; it breaks up a signal into shifted and scaled versions of the original wavelet. Shifting a wavelet means moving it forward or backward in time. Scaling a wavelet, on the other hand, means stretching or compressing it to obtain low and high frequency wavelets. Smaller scale factors correspond to more compressed wavelets and vice versa. In particular, the wavelet analysis gives good time resolution at high frequencies, while it shows good frequency resolution at low frequencies.

Based on the advantages mentioned before, the output signal of MEMS inertial sensors can be purified through three principle steps: firstly, the decomposition of signal is performed by choosing an appropriate wavelet. And soft thresholding method is used to eliminate wavelet coefficients which belong to noise component. After that, the signal can be reconstructed from the filtered wavelet coefficients with the inverse wavelet transform. Figure 3 illustrates the basic architecture of the wavelet analysis for MEMS-based inertial data.

\section{EXPERIMENTAL RESULTS AND DISCUSSIONS}

In order to carefully observe the linearity of MEMS inertial sensor module, a turntable is utilized to record data for linearity calibration, which is shown in Fig. 4. With the help of this turntable, the angular rate or tilt angle is controlled exactly

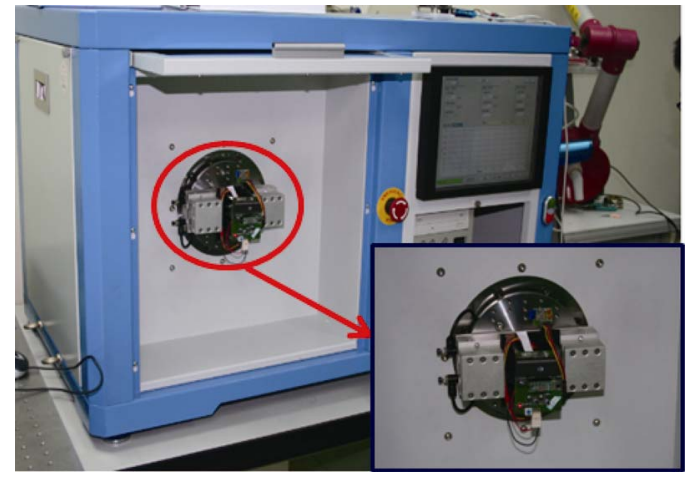

Fig. 4. Experimental equipment for MEMS inertial sensors calibration.

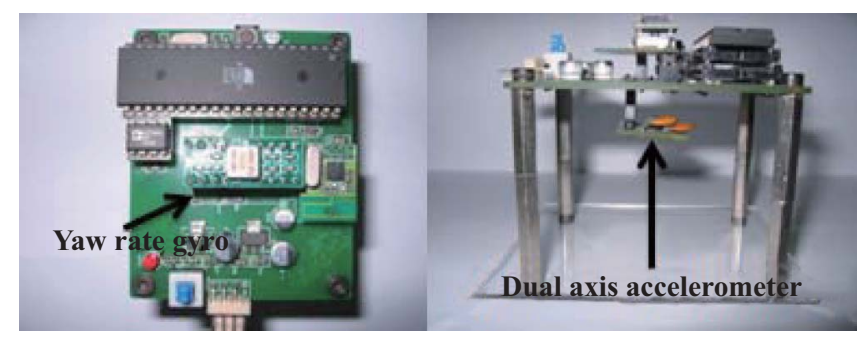

Fig. 5. MEMS inertial sensor module.

to obtain a reference rate or angle for linearity calibration. The MEMS inertial sensor module integrated accelerometer (ADXL213) and gyroscope (ADXRS150) as shown in Fig. 5. This unit was built as a low cost alternative using MEMS inertial sensor module. The output signals of MEMS inertial sensors are transmitted and received by a couple of RF ICs and displayed on our program developed by LabVIEW software. To begin with, the setting is at regular room temperature and hence temperature effect could be neglected. Then an experiment is carried out by recording the MEMS inertial sensor module output including accelerometer and gyroscope at rest and the result are shown in Fig. 6. An ideal bias output should be closed to a constant for a long time.

To cope with linearity calibration, the nonlinearity error is evaluated while the bias is measured previously. As shown in Fig. 7, for both accelerometer and gyroscope, the differences between ideal input and measured output are inconsistent, especially for lower accelerations or angular rates. This phenomenon will contribute an asymmetrical calculation when sensors are implemented to navigation or positioning system. According to the procedure mentioned above, accelerometer data is sampled by tilting it from $\pm 1 \mathrm{~g}$ with several steps. As for gyroscope, the output is collected from \pm 150 $\mathrm{deg} / \mathrm{sec}$ with $5 \mathrm{deg} / \mathrm{sec}$ step. The calibrated characteristic equation fitted to the data for accelerometer and gyroscope is obtained as shown in (4) and (5).

$$
y_{\text {accel }}=8.9548 a-0.0863
$$




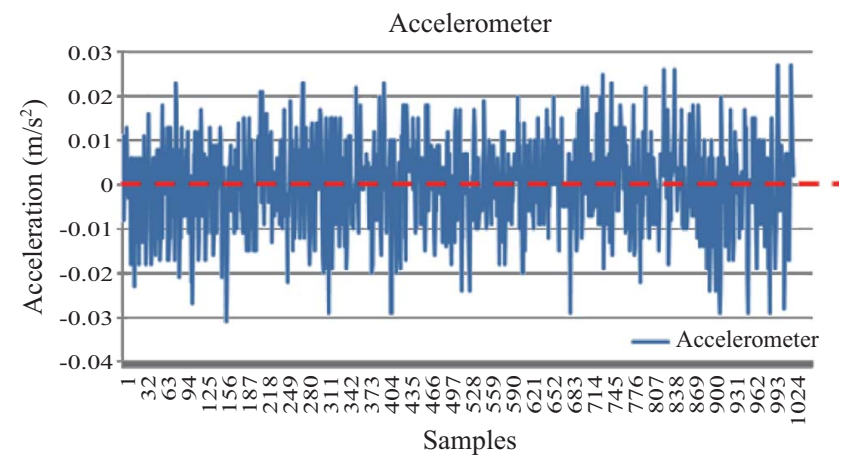

(a) Bias of accelerometer

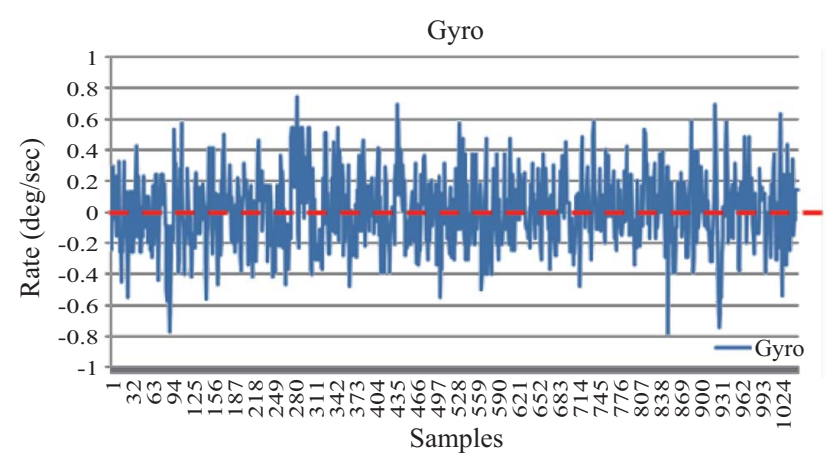

(b) Bias of gyroscope

Fig. 6. Static output of MEMS inertial sensor module.

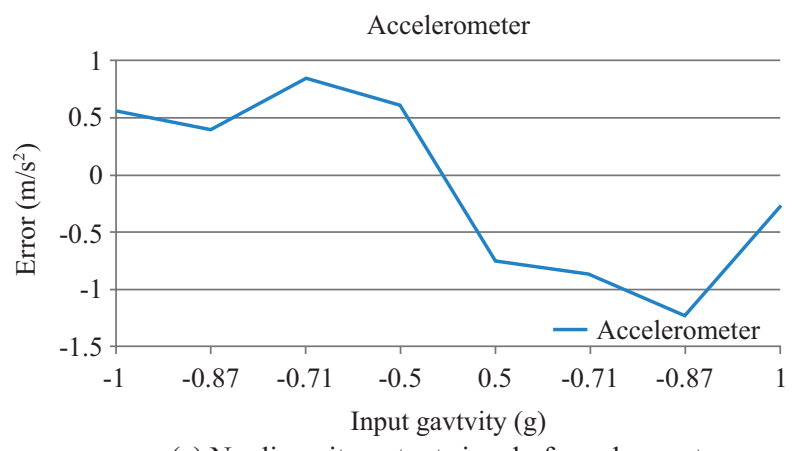

(a) Nonlinearity output signal of accelerometer

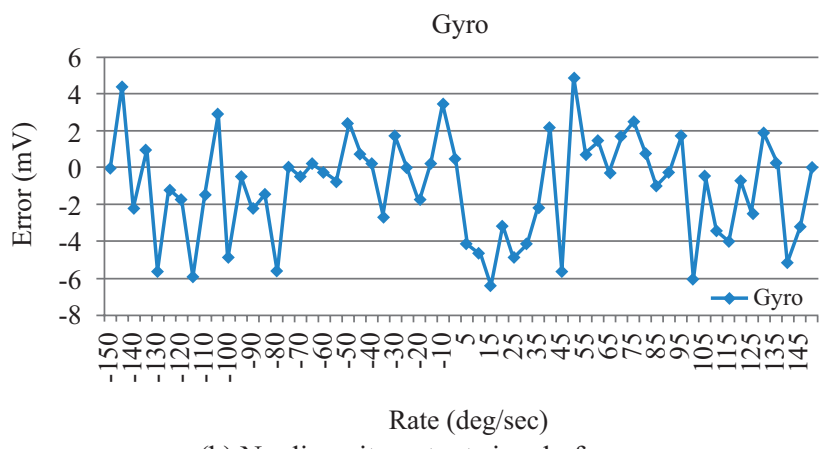

(b) Nonlinearity output signal of gyroscope

Fig. 7. Nonlinearity result of inertial sensors.

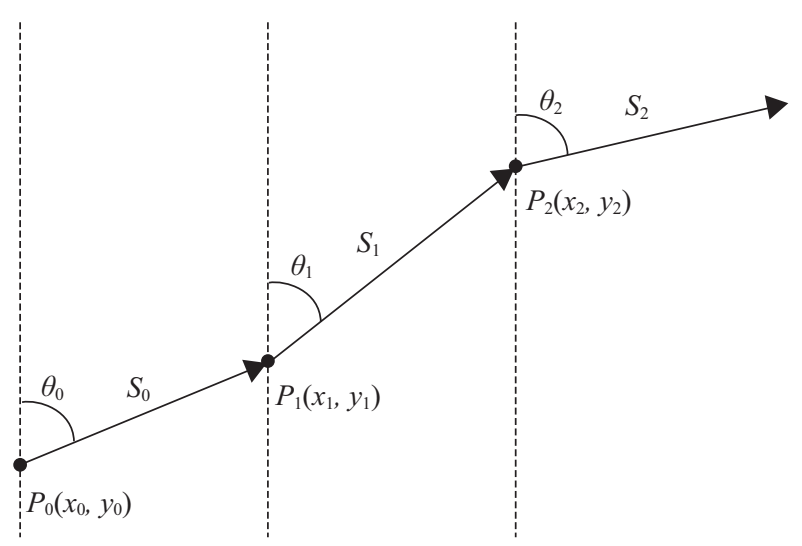

Fig. 8. The illustration of dead reckoning.

$$
y_{\text {gyro }}=0.0086 \omega+1.0062
$$

A trajectory examination for the MEMS inertial sensor module is then carried by moving along a $30 \times 40 \mathrm{~cm}$ rectangular. Figure 8 illustrates the principle of dead reckoning method to calculate the practical trajectory. Dead reckoning is the process of estimating current position by projecting heading angle and speed from a previous position, and the accumulated position is calculated as shown in (6) and (7). Obviously, an accelerometer and gyroscope will introduce an error proportional to $t^{2}$ in the position and the elapsed time $t$ respectively, as shown in (8) and (9). That is, the errors will be accumulated rapidly through numerical integration for underwater navigation or positioning.

$$
\begin{gathered}
x_{n}=x_{0}+\sum_{t=0}^{k-1} S_{t} \sin \theta_{t} \\
y_{n}=y_{0}+\sum_{t=0}^{k-1} S_{t} \cos \theta_{t} \\
P_{\text {meas }}=\iint a_{\text {meas }}=\frac{1}{2} a_{\text {meas }} \times t^{2} \\
\theta_{\text {meas }}=\int \omega_{\text {meas }}=\omega_{\text {meas }} \times t
\end{gathered}
$$

It deserves to be mentioned that it should be zero while the accelerometer or gyroscope is immobile. Figure 9 shows output signals of the MEMS inertial sensor module at rest for continuously 3 minutes. Clearly, the displacement increases quickly when it is in stationary. The accelerometer for moving forward about $50 \mathrm{~cm}$ is shown in Fig. 10. As we could see 


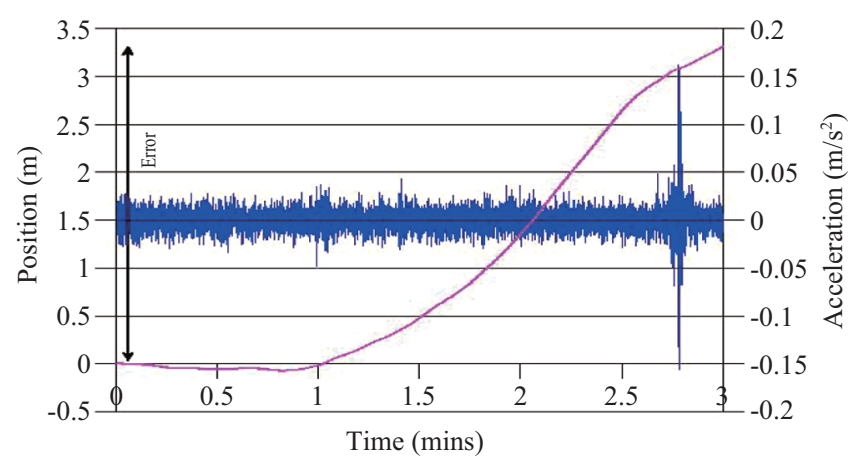

(a) Accelerometer signal and displacement

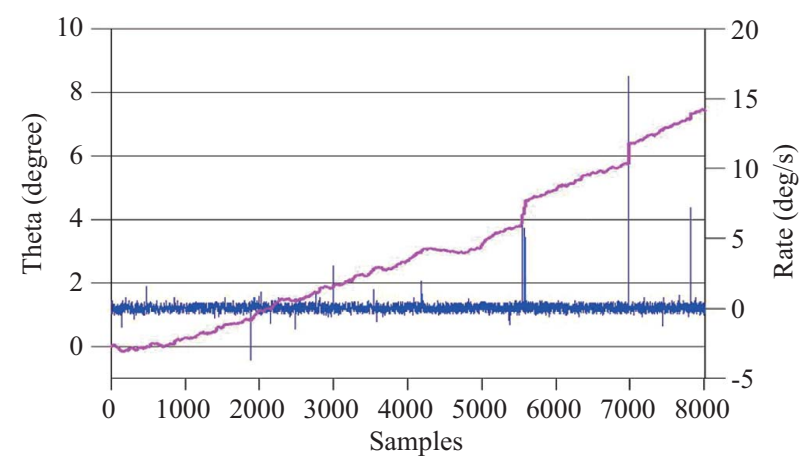

(b) Gyroscope signal and angle

Fig. 9. MEMS inertial sensors at rest.

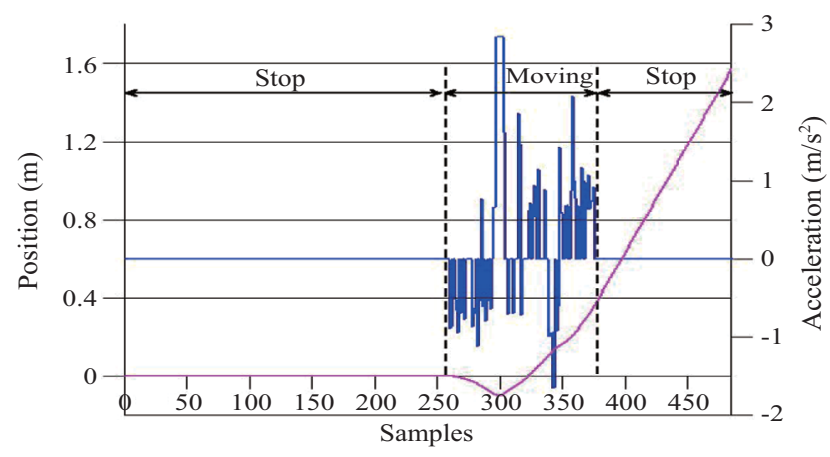

Fig. 10. Accelerometer and displacement for a forward trip.

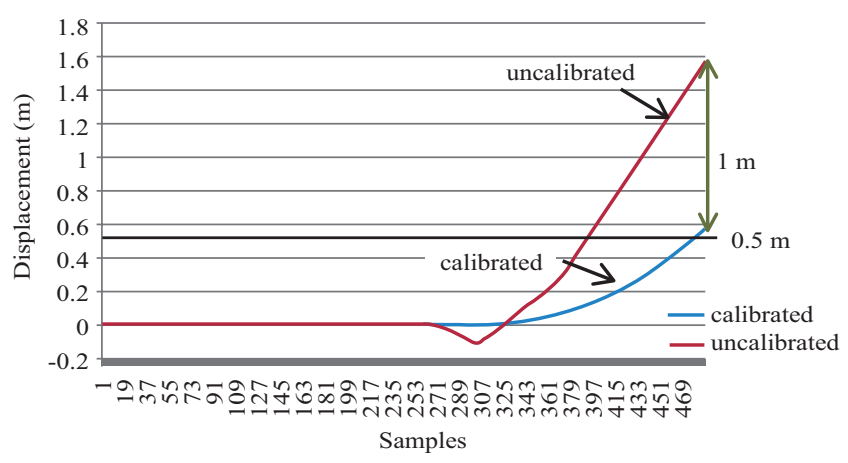

Fig. 11. The comparison of displacement result after calibration.

from the result, the errors of accelerometer cause an inaccuracy in the position determination. The calibrated displacement result is shown in Fig. 11. There is an improvement in the displacement error reduction and the deviation is about 10 $\mathrm{cm}$ compared to almost $1.6 \mathrm{~m}$ in the uncalibrated case. On the other hand, a result for rotating the gyroscope 90 degrees is shown in Fig. 12. In this result, the error between desired and practical angle is less than the case for accelerometer, but it has still a measurement error more than 10 degrees. And Fig. 13 shows that the rotation angle is close to 90 degrees after calibration. As a result, this new calibration method can

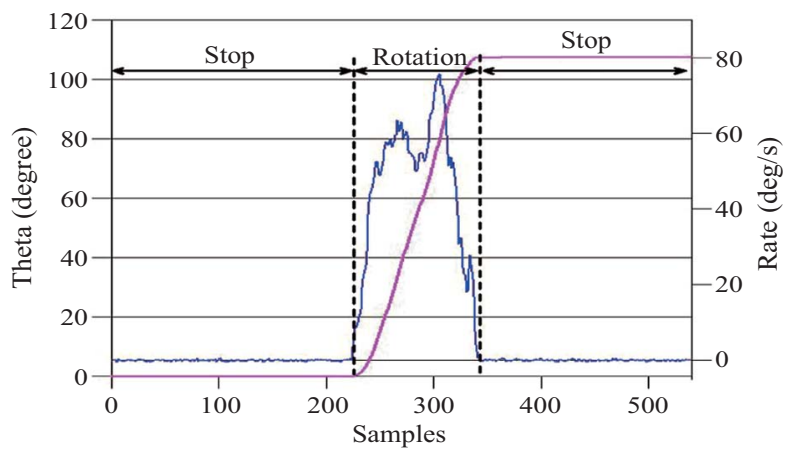

Fig. 12. The gyroscope and angle for a 90 degrees rotation.

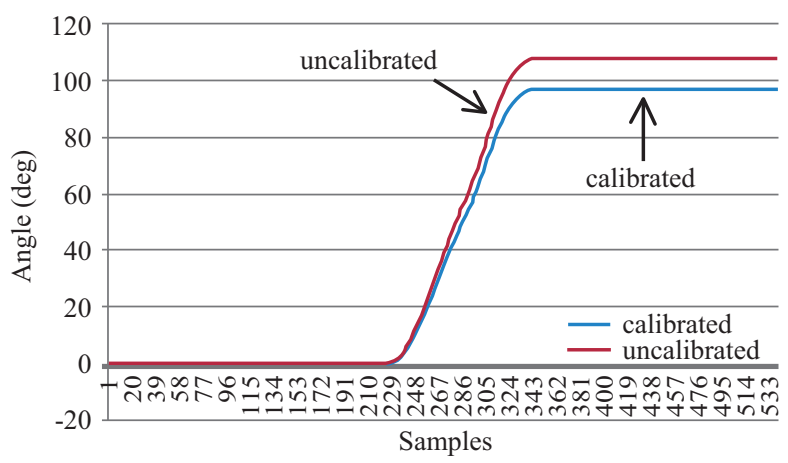

Fig. 13. The comparison of angle result after calibration.

be effective to enhance the accuracy and performance of MEMS inertial sensor module.

In the experiment of estimate trajectory, Fig. 14 is the estimated trajectory with only using numeric integration for moving along a $30 \times 40 \mathrm{~cm}$ rectangular. This result shows that the cumulative position diverges quickly compared to the original trajectory. Figure 15 is a comparison between the original and the practical trajectory when the calibration method is applied. This outcome indicates that there is improvement in the error reduction with the aid of the proposed calibration method. 


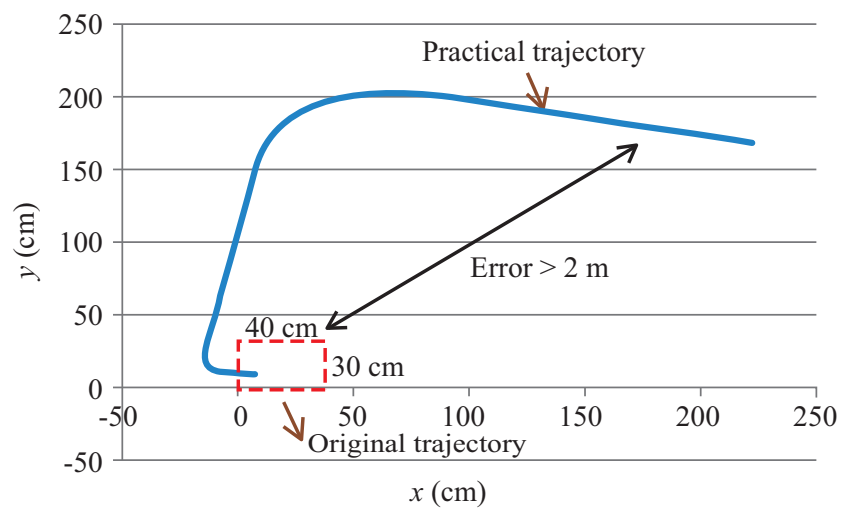

Fig. 14. The trajectory estimation without calibration.

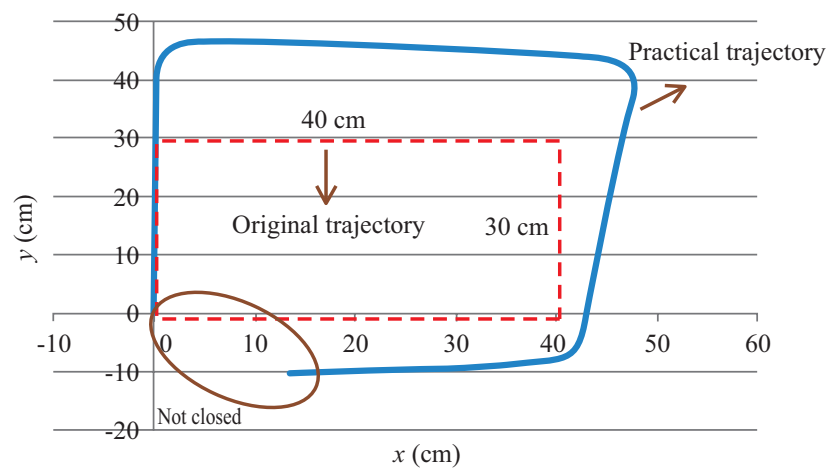

Fig. 15. The trajectory estimation with calibration.

\section{CONCLUSION}

This paper presents a calibration method for MEMS inertial sensors to improve the performance and accuracy. It is developed based on the relationship of measurement and physical input. Through this paper, it is shown that MEMS inertial sensors indeed suffer from large errors which degrade the practicability. Further, the performance of this proposed calibration method is evaluated by a trajectory test. The experimental result shows that this calibration method is useful to decrease errors in positioning calculation. However, the estimated trajectory is not close to the starting point. This is because the motion signals sensed by MEMS inertial sensor module belong to lower frequency range mainly, and it is difficult to distinguish between the real motion signals and noise. Besides, the performance and processing speed of computer should maintain stable for data acquisition to receive complete inertial data.

\section{ACKNOWLEDGMENTS}

The authors would like to thank National Science Council (NSC) for their financial supports to the project (granted number: NSC 97-2628-E-006-124-MY2).

\section{REFERENCES}

1. Aggarwal, P., Syed, Z., and El-Sheimy, N., "Thermal calibration of low cost MEMS sensors for land vehicle navigation system," Proceeding of the Vehicular Technology Conference, Singapore, pp. 2859-2863 (2008).

2. Bang, W.-C., Chang, W., Kang, K.-H., and Choi, E.-S., "Self-contained spatial input device for wearable computers," Proceeding of Seventh IEEE International Symposium on Wearable Computers, White Plains, NY, USA, pp. 26-34 (2003).

3. Chae, J.-S., Kulah, H., and Najafi, K., "An in-plane high-sensitivity, low-noise micro-g silicon accelerometer with CMOS readout circuit," The Journal of Microelectromechanical Systems, Vol. 13, pp. 628-653 (2004).

4. Djuric, Z., "Mechanisms of noise sources in microelectromechanical systems," The Journal of Microelectronics Reliability, Vol. 40, pp. 919932 (2000).

5. El-Diasty, M., EI-Rabbnay, A., and Pagiatakis, S.," Temperature variation effects on stochastic characteristics for low-cost MEMS-based inertial sensor error," The Journal of Measurement Science and Technology, Vol. 18, pp. 3321-3328 (2007).

6. El-Rabbany, A. and El-Diasty, M., "An efficient neural modal for denoising of MEMS-based inertial data," The Journal of Navigation, Vol. 57, pp. 407-415 (2004).

7. Gaysse, J., "A low cost absolute position calculation system," Proceeding of SICE-ICASE International Joint Conference, Busan, Korea, pp. 56585661 (2006).

8. Grewal, M. S., Henderson, V. D., and Miysako, R. S., "Application of Kalman filtering to the calibration and alignment of inertial navigation systems," IEEE Transactions on Automatic Control, Vol. 36, pp. 3-13 (1991).

9. Hide, C., Moore, T., and Smith, M., "Adaptive Kalman filtering for low-cost INS/GPS," The Journal of Navigation, Vol. 56, pp. 143-152 (2003).

10. Nelles, O., Nonlinear System Identification, Springer, New York, pp. 3566 (2001).

11. Nikbakht, N., Mazlom, M., and Khayatian, A., "Evaluation of solid-state accelerometer for positioning of vehicle," Proceeding of the IEEE International Conference on Industrial Technology, Hong Kong, pp. 729733 (2005).

12. Niu, X., Goodall, C., Nassar, S., and El-Sheimy, N., "An efficient method for evaluating the performance of MEMS IMUs," Proceeding of IEEE/ ION Position, Location, and Navigation Symposium, San Diego, California, pp. 766-771 (2006).

13. Pang, G. and Liu, H., "Evaluation of a low-cost MEMS accelerometer for distance measurement," The Journal of Intelligent and Robotic Systems, Vol. 30, pp. 249-265 (2001).

14. Park, M. and Gao, Y., "Error analysis and stochastic modeling of low-cost MEMS accelerometer," The Journal of Intelligent and Robotic Systems, Vol. 46, pp. 27-41 (2006).

15. Pastia, L., Walczak, B., Massant, D. L., and Reschiglian, P., "Optimization of signal denoising in discrete wavelet transform," The Journal of Chemometrics and Intelligent Laboratory Systems, Vol. 48, pp. 21-34 (1999).

16. Shkel, A., Howe, R. T., and Horowitz, R., "Modeling and simulation of micromachined gyroscopes in the presence of imperfections," Proceeding of the International Conference on Modeling and Simulation of Microsystems, San Juan, Puerto Rico, pp. 605-608 (1999).

17. Wang, H. and Tian, W., "Modeling the random drift of micro-machined gyroscope with neural network," Neural Processing Letters, Vol. 22, pp. 235-247 (2005).

18. Zhou, J. and Bolandhemmat, H., "Integrated INS/GPS system for an autonomous mobile vehicle," Proceedings of the IEEE International Conference on Mechatronics and Automation, Harbin, China, pp. 694-699 (2007). 